\title{
A Novel Business Process Prediction Model Using a Deep Learning Method
}

\author{
Nijat Mehdiyev $\cdot$ Joerg Evermann $\cdot$ Peter Fettke
}

Received: 31 October 2017/ Accepted: 22 May 2018/Published online: 19 July 2018

(C) Springer Fachmedien Wiesbaden GmbH, part of Springer Nature 2018

\begin{abstract}
The ability to proactively monitor business processes is a main competitive differentiator for firms. Process execution logs generated by process aware information systems help to make process specific predictions for enabling a proactive situational awareness. The goal of the proposed approach is to predict the next process event from the completed activities of the running process instance, based on the execution log data from previously completed process instances. By predicting process events, companies can initiate timely interventions to address undesired deviations from the desired workflow. The paper proposes a multi-stage deep learning approach that formulates the next event prediction problem as a classification problem. Following a feature pre-processing stage with n-grams and feature hashing, a deep learning model consisting of an unsupervised pre-training component with stacked autoencoders and a supervised fine-tuning component is applied. Experiments on a variety of business process log datasets show that the multi-stage deep learning approach provides
\end{abstract}

Accepted after one revision by Jelena Zdravkovic.

N. Mehdiyev $(\varangle) \cdot$ P. Fettke

Institute for Information Systems (IWi), German Research

Center for Artificial Intelligence (DFKI), Campus D3.2,

66123 Saarbruecken, Germany

e-mail: nijat.mehdiyev@iwi.dfki.de

P. Fettke

e-mail: peter.fettke@iwi.dfki.de

N. Mehdiyev · P. Fettke

Saarland University, 66123 Saarbruecken, Germany

J. Evermann

Memorial University of Newfoundland, 310 Elizabeth Avenue,

St. John's, NL A1B 3X5, Canada

e-mail: jevermann@mun.ca promising results. The study also compared the results to existing deep recurrent neural networks and conventional classification approaches. Furthermore, the paper addresses the identification of suitable hyperparameters for the proposed approach, and the handling of the imbalanced nature of business process event datasets.

Keywords Process prediction - Deep learning - Feature hashing $\cdot \mathrm{N}$-grams $\cdot$ Stacked autoencoders

\section{Introduction}

High-performance business processes are one of the last points of differentiation (Davenport and Harris 2007). Embedding predictive analytics into enterprise processes can boost business value (LaValle et al. 2011). Process aware enterprise information systems (EIS) such as workflow management systems (WMS), enterprise resource planning (ERP), customer relationship management (CRM), or incident management (IM) generate log events during process execution (van der Aalst et al. 2011). Such $\operatorname{logs}$ are a source for predictive analytics, which aids decision making by providing insights into future process behavior. An effective design and implementation of predictive approaches ensure that business activities will run in a desired manner by avoiding predicted failures and deviations from the intended process behavior. Detecting process anomalies in real-time, analyzing behavioral patterns of customers to make tailored offers, risk management by predicting compliance violations, or effective resource allocation, are some of the use cases of data driven predictive process analytics (Evermann et al. 2017).

Current EIS focus on enhancing a company's ability to achieve high-performing business processes. However, 
their effectiveness is limited by their lack of advanced predictive analytics. The built-in business intelligence solutions mainly address descriptive, such as demographic and performance problems. However, simply making operations more efficient is not enough for firms to remain competitive. They face challenges to transform the vast amount of generated data into smart decisions to deliver better products and services (Duan and $\mathrm{Da} \mathrm{Xu}$ 2012). Hence, future EIS need to shift from diagnostic examination of historical data to proactive decision making using predictive analytics. Predictive capabilities need to be embedded into the business processes. As process orchestration tools, EIS provide the necessary basis for this. Integrating advanced analytics with EIS is an important emerging trend in IS research (Sun et al. 2015).

Business process prediction predicts a target variable of interest after extracting features from business process $\log$ data. Predicting continuous target values, such as remaining process execution time, are regression problems. Predicting discrete target values, such as the next events in the running case, the outcome of a process instance, or the violation of service level agreements, are classification problems. In this study, we focus on predicting the next business process event, considering the past events of the running process instance, based on execution $\log$ data from previously completed process instances. This is an important problem in process analytics as such analytical information allows analysts to intervene proactively to prevent undesired behavior. We address this problem with a multi-stage deep learning approach. The main contribution of our research is threefold:

1. This study applies, for the first time in the business process management domain, a deep learning approach consisting of an unsupervised pre-training stage with stacked autoencoders, and a supervised fine-tuning stage for the multi-class classification problem. By initializing the parameters in all neural networks layers using greedy layerwise pre-training with autoencoders, followed by a minimization of a global training criterion using labels, we improve on current process prediction methods.

2. This study improves on prior research by incorporating an extensive data pre-processing stage. We use an n-gram representation and feature hashing approach to build numerical feature vectors from event log data. To our knowledge, no prior studies have applied feature hashing in this domain. Encoding process data so as to take into consideration their sequential nature, and reducing the dimensionality of this encoding to speed up the inference process of the deep neural networks, are crucial tasks that were examined carefully in our study.

3. We address the hyperparameter optimization of our deep learning approach, and the imbalanced nature of the process data to further improve prediction precision.

We follow the "exaptation" (extend known solutions to new problems) type of design science research (DSR) knowledge contribution by adopting successful solutions (stacked autoencoders based deep learning, feature hashing) to build innovative predictive analytics models for process data in EIS (Gregor and Hevner 2013).

The remainder of the paper is organized as follows: Sect. 2 introduces related work on business process prediction. Section 3 provides a broad description of the components of the proposed approach. It discusses the data pre-processing stages, $n$-gram encoding and feature hashing, and the structure of the deep learning model. Section 4 outlines the experimental settings, the structure of datasets and our empirical results. Section 5 concludes the paper with a discussion and summary.

\section{Related Work}

A growing body of literature has examined machinelearning approaches in business process management. We categorize them according to the type of the target variable (discrete vs. continuous) they predict, and discuss the problem types within these categories.

The first category comprises approaches that deal with regression problems. Predicting the remaining processing time of incomplete cases is the most frequently addressed problem in this category. van Dongen et al. (2008) applied non-parametric regression approaches to compute the remaining cycle time on the data recorded in event logs. Polato et al. (2016) implemented both simple and support vector regression methods to forecast the remaining time of running process instances. Rogge-Solti and Weske (2013) proposed a stochastic Petri net with generally distributed transitions to predict remaining process execution time based on elapsed time since the last observed event. To overcome the shortcomings of conventional regression approaches in predicting remaining time to completion, van der Aalst et al. (2011) presented an annotated transition system that represents an abstraction of the process with time annotations. Folino et al. (2012) introduced a hybrid predictive clustering tree (PCT) and multiple performance annotated finite state machine (FSM) models for remaining time prediction. Senderovich et al. (2017) applied linear regression, random forests and XGBoost approaches for remaining time prediction after obtaining the features related from specific process instances and global process models.

The second category deals with classification problems, including process outcome predictions, service level 
agreement violations, nominal attribute prediction, next event prediction etc. (Kang et al. 2012a, b; Leontjeva et al. 2015; Metzger et al. 2015; Di Francescomarino et al. 2016). The following studies address the next process event prediction that we investigate in this paper. A multi-stage model, which starts by clustering event sequences using the k-mean algorithm combined with sequential alignment, builds individual Markov models on the obtained clusters (Le et al. 2014). Experiments were conducted on records of processes obtained from a telecommunication company. An approach by Le et al. (2017) uses sequential k-nearest neighbor classification and an extension of Markov models to predict the next process steps by considering temporal features. Using the same process log data as Le et al. (2014), they showed the superiority of this model over Markov and Hidden Markov Models (HMM). Unuvar et al. (2016) proposed a decision tree model to predict the next activity in running instances of processes with parallel execution paths. Five different models for representing the path attribute of the execution trace were presented and experiments were conducted on simulated data. Combining the two approaches yields a hybrid model, which learns a decision tree at each node of the process model, based on the execution traces to compute the transition probabilities, and creates a Markov chain model (Lakshmanan et al. 2015). A simulated dataset was used to verify the prediction accuracy. Somewhat similar to a Markov model, a probabilistic finite automaton (PFA) based on Bayesian regularization by Breuker et al. (2016) uses the expectation maximization (EM) approach to estimate the relevant process parameters. The evaluation was done using both simulated and real data (the publicly available BPI Challenge 2012 and BPI Challenge 2013 data). Márquez-Chamorro et al. (2017) proposed an evolutionary rule based approach to predict the events of interest after encoding the features using a window technique. The approach was evaluated using the BPI Challenge 2013 and health services datasets.

Recent work is moving from explicit process models to deep learning approaches. Evermann et al. (2017) applied recurrent neural networks (RNN) with long short-term memory (LSTM) after transforming the input features using word embeddings. They also investigated accuracy improvement due to adding available case and event specific explanatory variables. BPI Challenge 2012 and 2013 datasets were used to validate the prediction results. Also applying the LSTM approach, but only considering the occurrence sequence of the activities and their timestamps, Tax et al. (2017) transformed the input activities to feature vectors using one-hot encoding. Both studies examined the prediction of process activity duration using the same approach. Our own earlier, initial study is also based on a deep learning approach (Mehdiyev et al. 2017).
However, this paper significantly expands on the earlier paper by improving the hyperparameter optimization, assessing and improving prediction performance on imbalanced datasets (which are typically problematic for classifiers), and additional evaluation and comparison.

One of the main differences between the studies by Evermann et al. (2017) and Tax et al. (2017) and our approach lies in the transformation of the sequential process data to the neural network input features (for the predetermined prefix size). Most existing approaches use the simple index encoding method to build a feature vector from sequence data, but this does not consider the interdependencies among the sequential event data (Leontjeva et al. 2015; Márquez-Chamorro et al. 2017; Senderovich et al. 2017). To tackle this problem, we use an n-gram based encoding schema. Depending on the size of the event space, the n-gram based approach can lead to a very high dimensional feature space. Therefore, we apply a feature hashing technique to obtain a reasonable input vector size. Another important feature of our study, which significantly improves on our approach in Mehdiyev et al. (2017), is the optimization of the deep learning hyperparameters, which has not been previously used in business process event prediction. Since the hyperparameter configuration significantly affects the classification results, testing models with only a few hyperparameter combination variations (manual search) is likely to lead to suboptimal results. Finally, no study except Márquez-Chamorro et al. (2017) addresses the classification problem for an imbalanced dataset. Identification of rare events can have important business implications. We address this problem by synthesizing new instances for the minority class using neural networks and thereby balancing the training data set.

\section{Proposed Approach}

We formulate the prediction of the next process event as a classification problem. Figure 1 shows an overview of our approach. After a data pre-processing stage, we apply deep learning algorithms on a feature matrix extracted from the control flow, data flow, resource, and organizational perspectives. Our approach starts with process events (control flow) obtained from event log data with a sliding window technique and encoded in letters into the n-gram feature representation (see the Fig. 1). Next, feature hashing maps the n-grams to hash keys. The hashed feature matrix is then extended by adding data and resource features. Once the extended feature matrix is available, the deep learning method is applied to predict the next process events. It consist of two components, an unsupervised layerwise pretraining component that produces higher level feature representations, and a supervised fine-tuning of the whole 


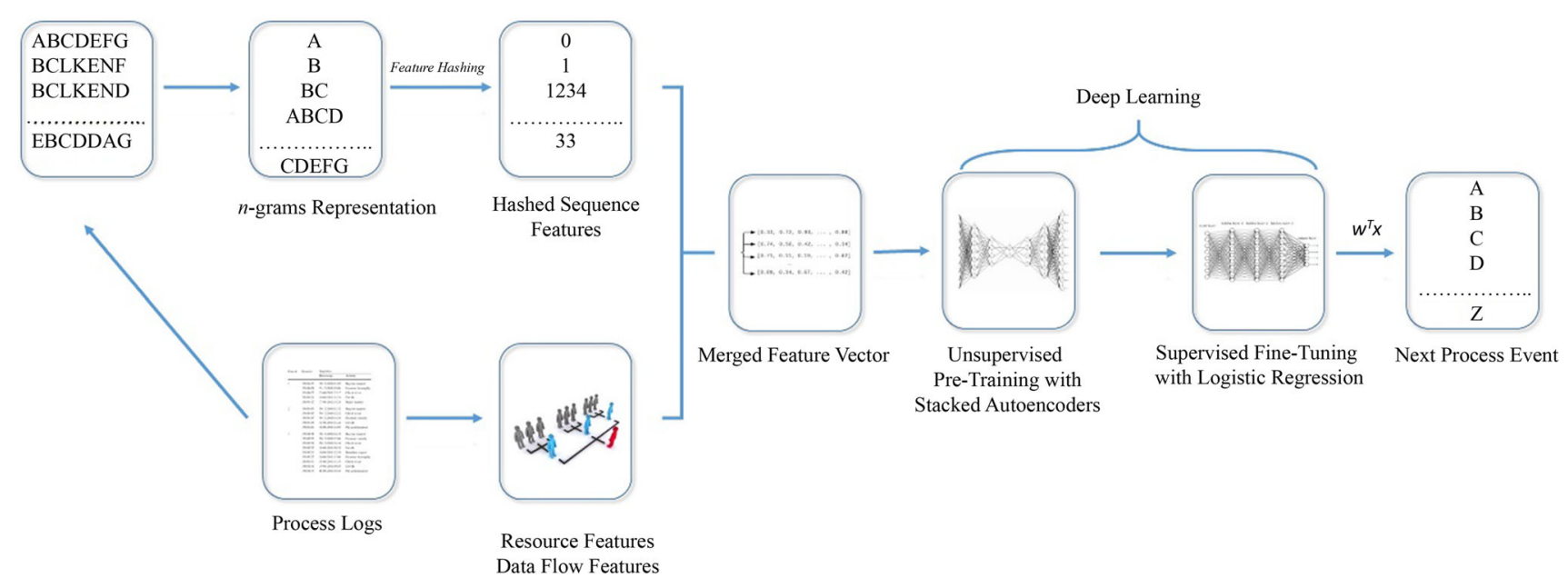

Fig. 1 The stages of the proposed approach

network for the multiclass classification, which adds an output layer on top of the stack.

\subsection{Terminology}

An event log consists of process traces. Each trace represents the execution of one process instance (case). A trace is sequence of events. Events contain attributes describing their characteristics (XES Standard 2016). Typical attributes are the name of the executing activity, the timestamp of the event, the lifecycle transition (e.g., "start" or "complete") and organizational resources or roles. Events are ordered by the timestamp of their occurrence. Other attributes may contain case specific information. The next event prediction problem is understood here as predicting the executing activity and lifecycle transition of the next event in the running trace, considering the sequence of past events for a predefined prefix length from that particular trace.

\subsection{Data Pre-processing}

Prior studies, with a few exceptions, pay little attention to data pre-processing. However, data preparation, comprising various stages such as data cleaning, encoding, dimensionality reduction, or feature extraction, significantly affects the predictive ability of classifiers.

\subsubsection{N-gram Encoding}

The first step of our approach is sequence encoding, converting character strings (specifically the executing activity for each event) into numerical input features. Leontjeva et al. (2015) provide a comparative analysis of various sequence encoding schemas for process outcome prediction. Choosing an appropriate encoding method is critical, as it significantly affects the accuracy of the machine learning approaches. Event sequence data contains intrinsic relationships and interdependencies among the events. We choose n-gram encoding as a suitable approach for modelling such dependencies due to its ability to represent relationships between neighboring elements by building all contiguous subsequences (Caragea et al. 2012). We use n-grams of different sizes, allowing us to extract both local and global features from the event sequences.

Definition 1 Given a sequence of events $E=$ $\left(e_{1}, e_{2}, \ldots, e_{N+(n-1)}\right)$ over the event universe $\varphi$, where the $N$ and $n$ are positive integers, an n-gram of $E$ is any $n$-long subsequence of consecutive events. There are $N$ such $n$ grams in $E$. The total number of possible unique n-grams for the event universe is $(|\varphi|)^{n}$ where the $|\varphi|$ is the total number of unique events in the process log data.

Assume that we have the sequence of events $\mathrm{E}=\{\mathrm{A}, \mathrm{F}, \mathrm{G}, \mathrm{C}, \mathrm{L}, \mathrm{B}, \mathrm{A}, \mathrm{D}, \mathrm{A}, \mathrm{M}\}$. The bigram (2-gram) features are all combinations such as $\{\mathrm{AF}, \mathrm{FG}, \mathrm{GC}, \ldots, \mathrm{AM}\}$; the trigram (3-gram) features are $\{$ AFG, FGC, GCL,$\ldots$, DAM $\}$ etc. We consider the combination of n-grams of pre-defined sizes. The size of our input feature space, e.g., in the case of 5-grams [including unigrams (1-grams), bigrams (2-grams), trigrams (3-grams), quadgrams (4-grams)] and an alphabet size of 15 unique events would be:

$N_{\text {total_features }}=15+15^{2}+15^{3}+15^{4}+15^{5}=813,615$

Due to its completeness (the alphabet is a priori known, in our case comprising the set of unique executing activities of the process events), domain independence, efficiency (one pass processing) and simplicity, the n-grams approach has been applied to various problems ranging from protein classification to information retrieval 
(Tomović et al. 2006). N-gram event data requires no additional preprocessing such as sequence alignment. The letter n-grams method is also very effective due to its ability to not only encode the letters but also order them automatically. However, as seen in the above example, the size of generated input feature set for classification problems tends to be very large: The number of features increases exponentially with the n-gram length. Using all generated features directly would lead to extremely high computational costs and the sparsity of the input would lead to reduced accuracy. To address this challenge, we adopt a dimensionality reduction technique, feature hashing, to reduce the size of n-gram feature vectors.

\subsubsection{Feature Hashing}

Feature hashing is an effective dimensionality reduction method that maps a high dimensional input space into a low dimensional space (Weinberger et al. 2009). Feature hashing has found successful applications in natural language processing (NLP), such as news categorization, spam filtering, sentiment analysis in social networks and different areas of bioinformatics (Forman and Kirshenbaum 2008; Ganchev and Dredze 2008; Caragea et al. 2012; Da Silva et al. 2014). The main idea of feature hashing is to use hash functions to map n-grams of events to feature vectors, which can be used to train the classifier.

Definition 2 Given a set of hashable features $N$, which are the n-grams obtained from the process event sequences, $h$ is the first hash function, $h: N \rightarrow\{1, \ldots . ., m\}$ and $\xi$ is the second hash function, $\xi: N \rightarrow\{ \pm 1\}$. The combined feature hashing function $\Phi^{(h, \xi)}$ maps the high dimensional input vector of size d into a low-dimensional feature vector $\boldsymbol{m}$ where $m<d$. The $i$ th element of the $\Phi^{(h, \xi)}(x)$ is given as: $\Phi_{i}^{(h, \xi)}(x)=\sum_{j: h(j)=i} \xi(j) x_{j}$ where $j=0, \ldots, d$ and $i=0, \ldots, m$.

Feature hashing not only reduces the training computational costs due to the reduced feature dimensionality but also conserves memory. However, dimensionality reduction via feature hashing can lead to information loss due to hash collisions, i.e. the mapping of many n-grams to the same hash keys. Larger hash tables, i.e. larger bit sizes of the hash function, can prevent this problem (Weinberger et al. 2009). Bit size determines the numbers of the bits when creating the hash table. The optimal bit size depends on the size of the n-gram vocabulary. A descriptive analysis of the n-grams obtained from the process sequences shows that they follow Zipf's law (Evermann et al. 2017). This implies that a small proportion of the input features occur with higher frequencies. Hence, hash collisions are likely to take place for infrequent variables and will incur low information loss (Caragea et al. 2012). The phenomenon can also be observed in protein sequence classification problems (Caragea et al. 2012). As a reasonable trade-off between dimensionality reduction and information loss, we use the 32 bit murmurHash function (Langford et al. 2007) as hash function $h$,. The binary hash function $\xi$ is included to ensure that the hash kernel is unbiased (Weinberger et al. 2009).

\subsection{Deep Learning Model}

Artificial neural networks (ANN) offer a number of advantages over alternative machine learning approaches for supervised learning tasks, including less need for formal statistical modelling, the ability to detect complex nonlinear relationships between predictors and outcomes, the ability to model the interrelationships among the predictor variables, and the availability of a range of training algorithms (Tu 1996). The superior performance of ANN has been documented in many comparative empirical studies and competitions (Caruana and Niculescu-Mizil 2006; Caruana et al. 2008; Schmidhuber 2015).

The traditional approach to train ANNs, particularly deep neural networks with multiple hidden layers, directly optimizes the loss function through stochastic gradient descent, beginning from randomly initialized weights. However, this results in long training durations and reduced prediction performance (Vincent et al. 2010). Beginning in the mid-2000s more effective training methods (Hinton et al. 2006; Vincent et al. 2008), such as deep belief network (DBN), and various autoencoder architectures have been developed. The training process for these network architectures consists of two stages: (1) unsupervised greedy, layerwise pre-training and (2) supervised fine-tuning. The main idea of the unsupervised pre-training is to address the need for learning complicated functions that represent high-level abstractions. Network weights are obtained through self-supervised learning that learns the non-linear transformation of the original input. The weights obtained from this stage are then used for training the whole network. The supervised fine-tuning component maps the output data to the pre-trained deep neural network and tries to minimize classification errors with gradientbased optimization by adjusting the previously learned weights.

An extensive experimental study showed that neural networks with unsupervised pre-training provide better classification results than networks without: The unsupervised pre-training yields a good initial marginal distribution, captures intrinsic dependencies between variables, outperforms classical regularization techniques, and acts as a variance reduction technique (Erhan et al. 2010). We apply stacked autoencoders to extract high-level feature 
Unsupervised Pre-training with Stacked Autoencoders

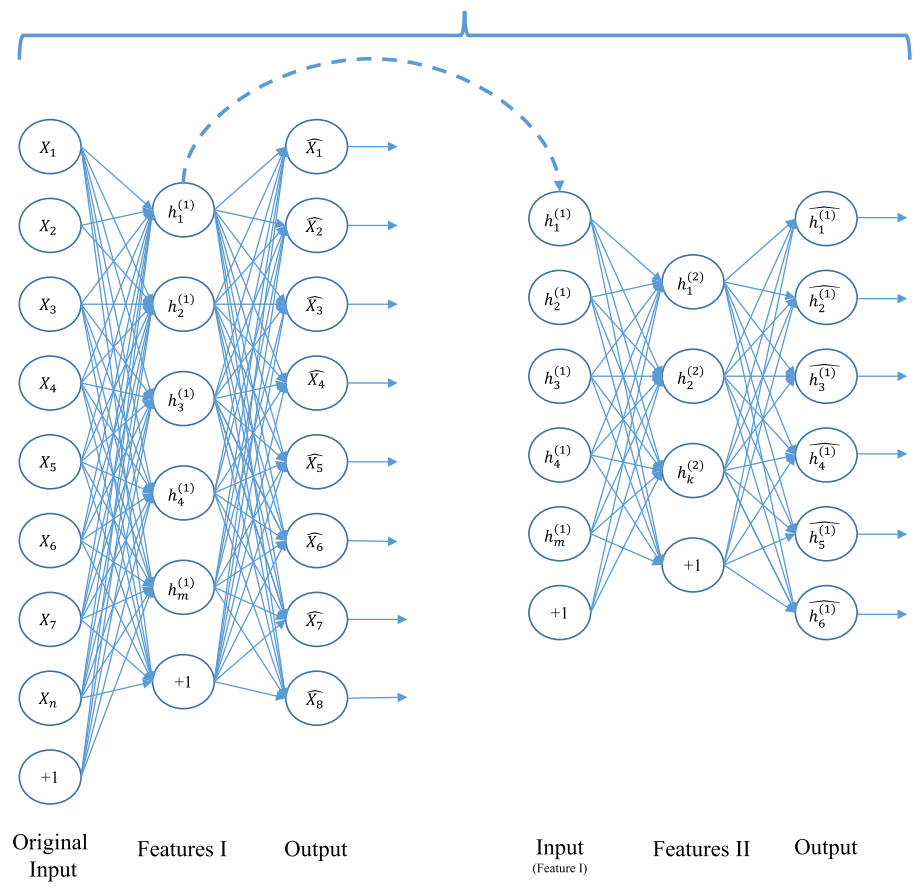

Supervised Fine-tuning with Logistic Regression

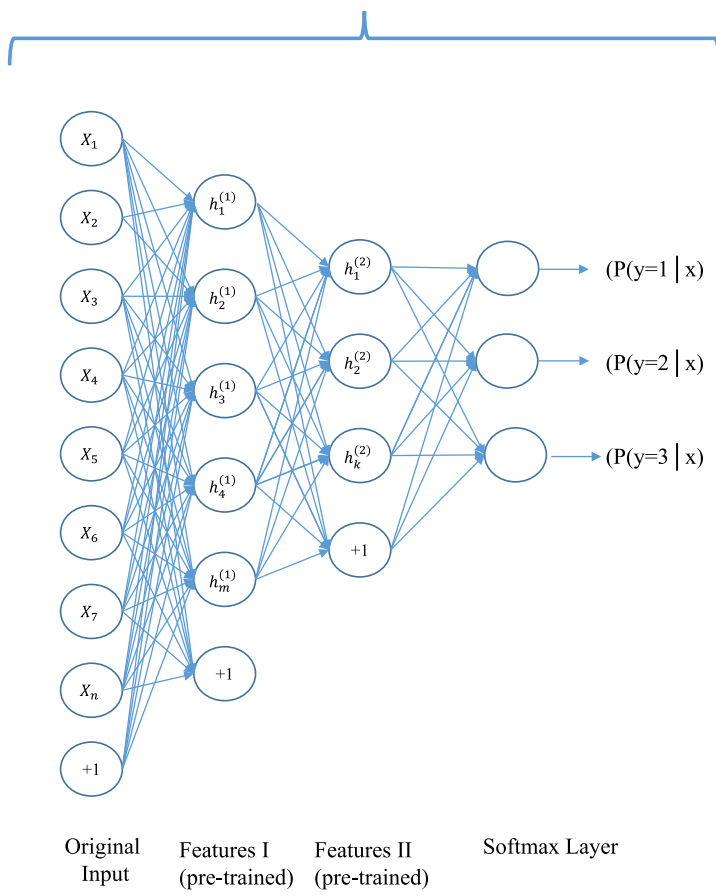

Fig. 2 Stacked autoencoders based deep learning. Unsupervised pre-training on the left, supervised fine-tuning on the right

representation layerwise in an unsupervised manner. After pre-training with stacked autoencoders, we perform the fine-tuning and relevant classifications using a logistic regression layer after adding an output layer to the obtained stack (see the Fig. 2).

\subsubsection{Unsupervised Pre-training with Stacked Autoencoders}

Autoencoders are the non-linear generalization of the principal component analysis (PCA) that can model nonlinear interdependencies among features (Hinton and Salakhutdinov 2006). An autoencoder consists of three layers, namely input, hidden and output layers. The hidden layer is referred to as encoding layer while the output layer acts as a decoding layer.

Encoder: The encoder maps the high-dimensional input vector $\mathrm{x} \in[0,1]^{\mathrm{d}}$ to the hidden layer using a non-linear activation function $f_{\theta}$. Due to its tendency to increase sparsity and reduced tendency of vanishing gradients (Izadyyazdanabadi et al. 2017; Shi and Chu 2017), we adopted the rectified linear unit (ReLU) as an activation function for encoding:

$h=f_{\theta}(x)=\sigma(W x+b)$

$\theta=\{W, b\}$ is the parameter set of the encoder where $W$ is a $d^{\prime} \times d$ weight matrix and $b$ is the bias. $h \in[0,1]^{\mathrm{d}}$ is the output of the hidden layer representation and $\sigma$ is the ReLU activation function.

Decoder: The decoder maps the hidden layer representation back to the reconstructed vector $\mathrm{z} \in[0,1]^{\mathrm{d}}$ through the mapping function $g_{\theta^{\prime}}$.

$z=g_{\theta^{\prime}}(h)=\sigma\left(W^{\prime} h+b^{\prime}\right)$

The main goal of the training is the optimization of parameter sets $\theta=\{W, b\}$ in the encoder and $\theta^{\prime}=\left\{W^{\prime}, b^{\prime}\right\}$ in the decoder to minimize the reconstruction loss. The squared error was used as the reconstruction loss function L:

$L(x, z)=\|x-z\|^{2}=\left\|x-\sigma\left(W^{\prime}(\sigma(W x+b))+b^{\prime}\right)\right\|^{2}$

This optimization problem was solved using the mini batch stochastic gradient descent method.

Stacked autoencoders is a greedy layer-wise approach which conducts multi-phase feature extraction by using the features extracted by one autoencoder, represented by its hidden layer, as input of another, following autoencoder (left side of Fig. 2) The stacked autoencoders are trained independently to obtain the initial weights for the next stage, supervised fine-tuning. In our study, we deploy an undercomplete autoencoder (a network architecture with decreasing width of hidden layers) to address the process prediction problem. 


\subsubsection{Supervised Fine-Tuning}

After unsupervised reconstruction based learning of the network weights, logistic regression is applied to fine-tune the weights after mapping the output to class labels (right side of Fig. 2). For this, the decoding parts of the stacked autoencoders are removed and the logistic regression layer is added on top of the trained encoding layers. Since we deal with a multi-class classification problem, the added layer uses Softmax (multinomial logistic regression) units to estimate the probabilities of the classes:

$P(y=j \mid x)=\frac{e^{\theta_{j}}}{\sum_{i=1}^{k} e^{\theta_{i}}}$

The probability of the target class $y$ being class $j$, given the input $x$, is calculated from the input vector $x$ and a set of weighting vectors $w_{j}$, where $\theta_{j}=w_{j}^{T} x$ denotes the inner product of $w_{j}$ and $x$. The combined network is trained using usual multi-layer perceptrons to minimize the prediction error. We use stochastic gradient descent (SGD) to minimize the cost function. A lock-free methodology was adopted to parallelize the SGD where the multiple cores contribute to gradient updates (LeCun et al. 2012; Goodfellow et al. 2013).

\section{Evaluation}

To gauge the effectiveness of the proposed deep learning, we conducted a range of experiments with different datasets, experimental settings and evaluation purposes. We investigated the following research questions:

- RQ1: Does the proposed multi-stage deep learning approach provide superior results for different evaluation measures compared to existing classification approaches?

- RQ2: Does the proposed multi-stage deep learning approach outperform the LSTM based approaches by Evermann et al. (2017) and Tax et al. (2017) and the probabilistic finite automaton (PFA) based on Bayesian regularization by Breuker et al. (2016)?

The third contribution of this study is to use data balancing to improve classification accuracy (cf. Sect. 1). Business processes may contain rare activities that are not on the typical execution path. This leads to imbalanced event logs, where some events are highly prevalent and others are only sparsely represented, which are a challenge for classifier training. However, rare activities are highly relevant in a business context as they may signal process exceptions, process escalation or compensation tasks. Hence, it is important for classifiers to correctly classify or
Table 1 Characteristics of dataset

\begin{tabular}{lcc}
\hline Datasets & \# of unique event types & \# of events \\
\hline BPI_2012_W_Completed & 6 & 72,413 \\
BPI_2012_A & 10 & 60,849 \\
BPI_2012_O & 7 & 31,244 \\
BPI_2013_Incidents & 13 & 65,533 \\
BPI_2013_Problems & 7 & 9011 \\
Helpdesk & 9 & 13,710 \\
\hline
\end{tabular}

predict rare events and we therefore ask the following research question:

- RQ3: Can process prediction with a multi-stage deep learning approach benefit from data balancing to improve the prediction performance for rare process events? Because traditional resampling techniques lead to overfitting, and the lack of information for costsensitive learning, we use radial basis function (RBF) neural networks to balance the data. The ability of this approach to enhance the classification performance has already been documented (Robnik-Šikonja 2014).

Our experiments were performed on an Intel i7-5500U $2.0 \mathrm{GHz}$ processor with $16 \mathrm{~GB}$ RAM. For data pre-processing, we used the dplyr package for $\mathrm{R}$ (Wickham and Francois 2015). We developed a Java-based application to build the n-grams from the process event data. Feature hashing was done on the Microsoft Azure ML platform using the Vowpal Wabbit library (Langford et al. 2007; Barga et al. 2015). Both the pre-trained, stacked autoencoders and the supervised deep learning part were created on the H20 open source deep learning platform (Candel et al. 2016). We used Weka (Hall et al. 2009) for experiments with traditional classifiers.

\subsection{Datasets}

The experiments used real-life datasets, the BPI Challenge 2012 (van Dongen 2012), BPI Challenge 2013 (Steeman 2013), and Helpdesk (Verenich 2016) data. Table 1 describes the datasets. The number of unique event types is the number of output classes in our multi-class classification problem.

The BPI Challenge 2012 dataset comprises 262,000 events for 13,087 cases, obtained from a Dutch financial institute. The activities related to a loan application process are categorized into three sub-processes: activities related to the application (A), activities belonging to applications (W) and activities related to the offer $(\mathrm{O})$. Events for the A and $\mathrm{O}$ sub-processes contain only the completion lifecycle transition, while the $\mathrm{W}$ process includes the scheduled, 
started and completed lifecycle transitions. As Evermann et al. (2017), Breuker et al. (2016), and Tax et al. (2017) use only the completion events, we remove the started and scheduled events from this sub-process. Similar to the previous papers, we evaluate our approach on three datasets from BPI Challenge 2012: BPI_2012_A, BPI_2012_O and BPI_2012_W_Completed.

The BPI Challenge 2013 dataset contains log data from an incident and problem management system of Volvo IT in Belgium. It has three subsets: The incident management subset encompasses 7554 cases with 65,533 events of 13 unique types. The open problems subset contains 819 cases with 2351 events of 5 unique types, and the closed problems subset comprises 1487 cases with 6660 events of 7 unique types. We merged the open and closed problems subsets to create a dataset identical to that in other studies, yielding 9011 events.

The helpdesk dataset comprises event data from a ticketing management system designed for the help desk of an Italian software company. The event log contains 3804 cases with 13,710 events.

The BPI Challenge 2012 data provides both organizational information such as the identification number of the resources initiating events, and case specific information such as the amount of the requested loan. The BPI Challenge 2013 datasets contain information about the priority of the problems and incidents, originating functional divisions and organizational lines, related products, process owners' countries and names. After generating the feature vectors from the sequence of the activities through $n$-grams and feature hashing approaches, we appended the additional information from the logs to the feature vector.

\subsection{Evaluation Metrics}

To evaluate the effectiveness of our deep learning approach and to compare it to other classification algorithms, we computed average accuracy, averaged precision, average recall, average F-measure, and Matthews correlation coefficient (MCC) and the area under the ROC curve (AOC) (see the Table 2), which were adapted to a multiclass classification problem.

In these formulas, $t p_{i}$ (true positives for class $i$ ) is the number of events of class $i$ that have been classified or predicted as being of class $i . f p_{i}$ (false positives) is the number of events not of class $i$ that have been classified (predicted) as being of class $i \cdot t n_{i}$ (true negative) is the number of events not of class $i$ that have been classified (predicted) as not of class $i$ and finally $f n_{i}$ (false negatives) is the number of events of class $i$ that have been classified (predicted) as not of class $i . t p r_{i}$ is the true positive rate and $f p r_{i}$ the false positive rate for class $i$. Accuracy is defined as the proportion of correctly predicted instances of all
Table 2 Evaluation metrics for multi-class classification

\begin{tabular}{ll}
\hline Metrics & Formula \\
\hline Accuracy & $\frac{1}{n} \sum_{i=1}^{l} s_{i} \frac{t p_{i}+t n}{t p_{i}+f n_{i}+t n_{i}+f p_{i}}$ \\
Precision & $\frac{1}{n} \sum_{i=1}^{l} s_{i} \frac{t p_{i}}{t p_{i}+f p_{i}}$ \\
Recall & $\frac{1}{n} \sum_{i=1}^{l} s_{i} \frac{t p_{i}}{t p_{i}+f n_{i}}$ \\
F-measure & $\frac{1}{n} \sum_{i=1}^{l} s_{i} \frac{\text { precision }_{i} \times \text { recall }_{i}}{\text { precision }_{i}+\text { recall }_{i}}$ \\
MCC & $\frac{1}{n} \sum_{i=1}^{l} s_{i} \frac{t_{i} \times p_{i} \times n_{i}-f p_{i} \times f n_{i}}{\sqrt{\left(t p_{i}+f p_{i}\right)\left(t p_{i}+f n_{i}\right)\left(t n_{i}+f p_{i}\right)\left(t n_{i}+f n_{i}\right)}}$ \\
AUC & $\frac{1}{n} \sum_{i=1}^{l} s_{i} \int_{0}^{1} t p r_{i} d\left(f p r_{i}\right)$ \\
\hline
\end{tabular}

1 is the number of classes, $s_{i}$ is the true size of class $i$ (the number of events of class $i$ ) and $n=\sum_{i=1}^{l} s_{i}$ is the total size of the dataset

instances. Precision determines how many activities were correctly classified for a particular class, given all predictions of that class. Recall is the true positive rate for a particular class. The F-measure is the harmonic weighted mean of precision and recall. MCC is referred as the correlation between the target values and predicted classifications. AUC is the area under the ROC (receiver operating characteristic) curve. We computed these measures for each individual class and obtained the overall value by summing up their scores, weighted by the true class size.

$80 \%$ of each dataset was used for training and $20 \%$ for testing. We used the training data for both unsupervised pre-training and supervised fine-tuning of our deep learning model. We used tenfold cross validation for training, in which the dataset was partitioned into the 10 disjoint subsets. Both training and validation were carried out 10 times. During each iteration, one subset was used for validating whereas the others were used for training the classifier. This procedure is important for finding the best hyperparameter configuration (Vincent et al. 2010). The test results were used to compare the approaches.

\subsection{Hyperparameter Optimization}

Deep neural networks may have more than fifty hyperparameter (Bergstra et al. 2011). Hyperparameter optimization significantly affects the learning process and prediction outcomes by identifying the best parameter configuration from the given hyperparameter space at a reasonable computational cost. In the traditional approach, manual search, experts define some hyperparameter values for different parameters based on their experience and intuitions (such as the number of hidden layers, the number of neurons, the learning rate etc.) and try to find the best combination of hyperparameter values by conducting multiple training sessions. Due to the time consuming 
Table 3 Optimal hyperparameter values for BPI Challenge 2012_A dataset

\begin{tabular}{llll}
\hline Parameters (pre-training) & Values & Parameters (whole network) & Values \\
\hline Number of neurons (hidden layers) & $425: 400: 390: 300$ & Number of layers & $6(4$ hidden) \\
Initial weight distribution & Normal distribution & Epochs & 100 \\
Sparse & True & Adaptive learning & True \\
Learn rate & 0.005 & Adaptive learning rate smoothing factor & $1 \mathrm{e}-8$ \\
Momentum & 0.9 & Adaptive learning rate time decay factor & 0.99 \\
Annealing rate & $10^{4}$ & Activation & ReLu \\
& & Activation (classification) & 20 \\
& & Batch size & 0 \\
& & classifier L2-penalty & Cross-entropy \\
& & Loss function & \\
\end{tabular}

nature of this approach, only a few hyperparameter value combinations can be tested (Bergstra et al. 2011). Furthermore, due to the shortcomings of human reasoning in multi-dimensional spaces, it is challenging to achieve globally optimal outcomes (Witt and Seifert 2017).

The brute force, exhaustive approach (grid search), trains the model for every possible combination of hyperparameter values by following some stopping criterion. Grid search identifies better hyperparameter configuration than manual search in the same computational time (Bergstra and Bengio 2012). Most deep learning studies use a combination of manual and grid search, where experts define the possible values for each variable and grid search then finds the best combination of these (Larochelle et al. 2007). Such an exhaustive search suffers from the "curse of the dimensionality" since the number of combinations increases exponentially with the number of hyperparameters (Bergstra et al. 2011). To address this, Bergstra and Bengio (2012) proposed a random search approach. The idea is to pick combinations of hyperparameter values randomly and to train the models in the given constraint (e.g., compute time). Empirical results show that random search outperforms the brute-force grid search (Bergstra and Bengio 2012).

Hence, we adopt the random search hyperparameter optimization approach. We defined the parameter ranges for number of hidden layers [3:10], number of neurons in the hidden layers [10:500] considering the undercomplete network structure, sparse data handling [True, False], initial weight distribution [uniform, normal] for the pretraining component, number of training epochs [10:1000], adaptive learning rate (adaptive learning rate time decay factor $=0.99$ and adaptive learning rate smoothing factor $=1 \mathrm{e}-8$ ), (initial) learning rate [0.0001:1], annealing rate $\left[10: 10^{6}\right]$ when adaptive learning is disabled, for both pre-training component and the whole network. We stopped the search when 200 models for a given dataset are trained. Training is stopped early if relative improvement is below a defined threshold. We used log-loss as the early stopping metric with a threshold of 0.001 . Table 3 shows the optimal hyperparameter configuration for the BPI_2012_A dataset. We performed hyperparameter optimization for all our experiments but do not show optimal values due to space restrictions.

\subsection{Results}

The following subsections provide discuss our experimental results to address our research questions. All reported results are from the test data subset.

\subsubsection{Comparative Analysis ( $R Q 1$ and $R Q 2$ )}

We first compared our approach to conventional (i.e. generic or not-process aware) classification algorithms including support vector machines (SVM), random forests, naïve Bayes, k-nearest-neighbours $(\mathrm{kNN})$ and $\mathrm{C} 4.5$ decision trees, which are among the most powerful and most widely-used algorithms (Wu et al. 2008). Table 4 presents our results for predicting the next event for prefix length 5, n-gram size 3 and bit size 10 .

The results for different performance measures show that, with few exceptions, our approach outperforms conventional, generic classification methods. The SVM method performs better than other methods over all three datasets and comes closest to our approach. For the BPI 2013 dataset, all methods except naïve Bayes perform similarly. The performance gaps between our approach and the alternative methods are quite large for the BPI 2012 and helpdesk datasets.

In summary, to answer RQ1, we observe that our proposed deep learning approach is superior to conventional, generic classification methods.

To examine RQ2, we compared our approach to three recent approaches for next event prediction. The results for all three BPI 2012 datasets show that our approach 
Table 4 Results obtained from conventional classification approaches and the proposed deep learning approach (higher numbers are better)

\begin{tabular}{lllllll}
\hline & Accuracy & Precision & Recall & F-score & MCC & AUC \\
\hline BPI 2012_A & & & & & & \\
SVM & 0.817 & $\mathbf{0 . 8 5 6}$ & 0.822 & $\mathbf{0 . 8 1 7}$ & 0.748 & 0.895 \\
RF & 0.720 & 0.714 & 0.721 & 0.712 & 0.566 & 0.888 \\
Naïve Bayes & 0.612 & 0.633 & 0.612 & 0.555 & 0.485 & 0.772 \\
C4.5 & 0.708 & 0.744 & 0.709 & 0.705 & 0.674 & $\mathbf{0 . 9 3 1}$ \\
Deep learning & $\mathbf{0 . 8 2 4}$ & 0.852 & $\mathbf{0 . 8 2 4}$ & $\mathbf{0 . 8 1 7}$ & $\mathbf{0 . 7 5 1}$ & 0.923 \\
BPI2013_Incidents & & & & & & \\
SVM & 0.652 & 0.599 & 0.653 & 0.622 & 0.350 & 0.730 \\
RF & 0.615 & 0.626 & 0.616 & 0.524 & 0.508 & 0.895 \\
Naïve Bayes & 0.576 & 0.618 & 0.577 & 0.590 & 0.519 & 0.879 \\
C4.5 & 0.659 & 0.558 & 0.659 & 0.582 & 0.564 & $\mathbf{0 . 9 0 0}$ \\
Deep learning & $\mathbf{0 . 6 6 3}$ & $\mathbf{0 . 6 4 8}$ & $\mathbf{0 . 6 6 4}$ & $\mathbf{0 . 6 4 7}$ & $\mathbf{0 . 5 8 3}$ & 0.862 \\
Helpdesk & & & & & & \\
SVM & 0.715 & 0.605 & 0.716 & 0.652 & 0.389 & 0.725 \\
RF & 0.601 & 0.619 & 0.601 & 0.606 & 0.278 & 0.688 \\
Naïve Bayes & 0.631 & $\mathbf{0 . 6 3 4}$ & 0.631 & 0.622 & 0.323 & 0.733 \\
C4.5 & 0.613 & 0.534 & 0.614 & 0.569 & 0.214 & 0.602 \\
Deep learning & $\mathbf{0 . 7 8 2}$ & 0.632 & $\mathbf{0 . 7 8 1}$ & $\mathbf{0 . 7 1 1}$ & $\mathbf{0 . 4 1 2}$ & $\mathbf{0 . 7 6 2}$ \\
\hline
\end{tabular}

outperforms all three approaches (see the Table 5). A bigger difference can be observed for the BPI_2012_W_Completed dataset, where our approach achieves an accuracy of 0.831 compared to 0.719 in Breuker et al. (2016) and 0.760 in Tax et al. (2017). The performance gap compared to Breuker et al. (2016) is greatest for recall (sensitivity). The comparison of our results with Evermann et al. (2017) in terms of precision also shows the superior performance of our proposed approach (0.811 vs. 0.658). Only two other studies used the BPI 2012_A and BPI_2012_O datasets to evaluate their models. Our approach outperforms both of those models in terms of all evaluation measures. The approach by Evermann et al. (2017) performs better for the latter two and achieves results close to ours.

The results for the BPI_2013_Incident dataset are mixed. The approach in Breuker et al. (2016) shows higher predictive performance than ours in terms of accuracy (0.714 vs. 0.663). However, our approach performs significantly better in terms of recall (0.664 vs. 0.377$)$. Precision results obtained in Evermann et al. (2017) are also better than for our approach. However, the experiments conducted on the BPI_2013_Problems dataset suggest that our approach delivers superior results compared to the alternatives.

Finally, only Tax et al. (2017) carried out experiments on the helpdesk data. Our approach performs better than LSTM approach in terms of accuracy (0.782 vs. 0.712$)$.

We also note that, since we use the random hyperparameter optimization instead of a manual search as in our previous study (Mehdiyev et al. 2017), the results presented here are a significant improvements over own earlier work (Mehdiyev et al. 2017), demonstrating the importance of this step.

In summary, to answer RQ2, we conclude that our approach outperforms existing deep-learning process prediction approaches for most datasets and on most quality metrics.

We examined the effect of the n-gram size on prediction accuracy. Since most process traces in the BPI_2012 and Helpdesk datasets contain less than 6 events, we defined the maximum length of prefix and n-grams as 5 . For the BPI_2013 we were able to build 10-grams. The experiment results suggest that increasing the size of the n-grams (from 2 to 5 and 10) does not lead to significant changes in the predictive capability of the model, while using longer n-grams increases computational costs. For example, the accuracy on the BPI2012_A and a prefix length of 5 ranges between 0.829 and 0.831 for $n$-grams sizes between 2 and 5, showing little improvement.

We also investigated the effect of the bitsize of the feature hashing on accuracy. As mentioned above, hash collisions can be reduced by increasing the bitsize of the hash table. Our results show that increasing the bitsize beyond a certain threshold does not improve prediction results. In our case, this threshold was 10 . This can be explained by the fact that the frequency of $n$-grams obtained from the process event sequences follow Zipf's law, which states that only a small proportion of the input features occur with higher frequencies (Caragea et al. 
Table 5 Comparison against benchmark approaches (higher numbers are better)

\begin{tabular}{|c|c|c|c|}
\hline & Accuracy & Precision & Recall \\
\hline \multicolumn{4}{|l|}{ BPI 2012_W } \\
\hline Breuker et al. (2016) & 0.719 & - & 0.578 \\
\hline Evermann et al. (2017) & - & 0.658 & - \\
\hline Tax et al. (2017) & 0.760 & - & - \\
\hline Proposed approach & 0.831 & 0.811 & 0.832 \\
\hline \multicolumn{4}{|l|}{ BPI2012_A } \\
\hline Breuker et al. (2016) & 0.801 & - & 0.723 \\
\hline Evermann et al. (2017) & & 0.832 & - \\
\hline Proposed approach & 0.824 & 0.852 & 0.824 \\
\hline \multicolumn{4}{|l|}{ BPI2012_O } \\
\hline Breuker et al. (2016) & 0.811 & - & 0.647 \\
\hline Evermann et al. (2017) & - & 0.836 & \\
\hline Proposed approach & 0.821 & 0.847 & 0.822 \\
\hline \multicolumn{4}{|l|}{ BPI2013_incidents } \\
\hline Breuker et al. (2016) & 0.714 & - & 0.377 \\
\hline Evermann et al. (2017) & - & 0.735 & \\
\hline Proposed approach & 0.663 & 0.648 & 0.664 \\
\hline \multicolumn{4}{|l|}{ BPI2013_Problems } \\
\hline Breuker et al. (2016) & 0.690 & - & 0.521 \\
\hline Evermann et al. (2017) & - & 0.628 & \\
\hline Proposed approach & 0.662 & 0.641 & 0.662 \\
\hline \multicolumn{4}{|l|}{ Helpdesk } \\
\hline Tax et al. (2017) & 0.712 & - & - \\
\hline Proposed approach & 0.782 & 0.632 & 0.781 \\
\hline
\end{tabular}

2012). This implies that the majority of hash collisions take place for infrequent, and thus less important, n-grams.

\subsubsection{Imbalanced Classification (RQ 3)}

In imbalanced datasets, some classes are severely underrepresented compared to others. This reduces the effectiveness of the machine learning techniques, especially for detecting the minority class examples (Wang and Yao 2012). To overcome this, various approaches at the data level (randomly or informatively under/over sampling), algorithm level, cost sensitive learning and boosting methods have been proposed (Sun et al. 2009). Due to their simple nature, resampling approaches are used frequently, but they are unable to increase the information that is required to train the models. Furthermore, undersampling may result in information loss. The SMOTE (Synthetic Minority Over-sampling Technique) method was proposed to address this issue. It generates new, non-replicated samples by interpolating neighboring minority class examples, but it also suffers from synthesizing noisy examples (Huang et al. 2016). Cost sensitive learning techniques are effective approaches to tackle the imbalanced classification problem but require cost information from domain experts. Huang et al. (2016) suggests that applying neural networks to synthesize the samples for minority class is a superior alternative.

In our study, we generate semi-artificial data of the minority class using radial basis function (RBF) neural networks (Robnik-Šikonja 2014). This approach extracts Gaussian kernels from the RBF trained with dynamic decay adjustment, and generates data from each kernel in the required proportions. Details and pseudo-code of the RBF based data generator can be found in Robnik-Šikonja (2014). We chose RBF networks for their advantages over other data generation methods. Although other methods consider the relationship between input and target variables, they do not consider dependencies among input variables. Such dependencies are preserved in the RBF based model. The RBF method assumes only the form of the data distribution (Gaussian), but uses extracted distribution parameters to generate data.

The process owners of the BPI Challenge 2013 Incidents dataset claim that employees try to find workarounds to stop the clock in order to manipulate the resolution time of an incident. Giving an incident a status of "Wait user" is one of these ways. Although employees were explicitly requested to avoid using the status of "Wait user" except for emergency cases, the guideline is occasionally broken. Identifying this misuse is therefore highly business relevant. However, this event occurs very infrequently. To handle this imbalanced classification problem, we reformulate the problem as a binary classification problem where the majority class is the set of all other events and the minority class is the "Wait user" event. We then apply our approach after balancing the class occurrence frequencies with the RBF method. We compare the results against the direct application of our approach to the imbalanced data (without rebalancing). Accuracy is inappropriate for comparing classification results for imbalanced datasets. Even when a classifier detects all majority examples correctly and fails to predict the examples from the minority class, the accuracy will still be high due to the prevalence of majority class examples (Han et al. 2005). Instead, we used the area under the ROC curve (AUC), which is an appropriate measure of the performance for imbalanced data (Bradley 1997). Figure 3 shows ROC curves for the imbalanced data and for the RBF rebalanced data.

The results show that balancing the dataset through RBF based data generation imrpoves the accuracy of our approach positively by increasing the AUC from 0.855 to 0.932 .

In summary, to answer RQ3, we conclude that RBF based data rebalancing works well in conjunction with our 


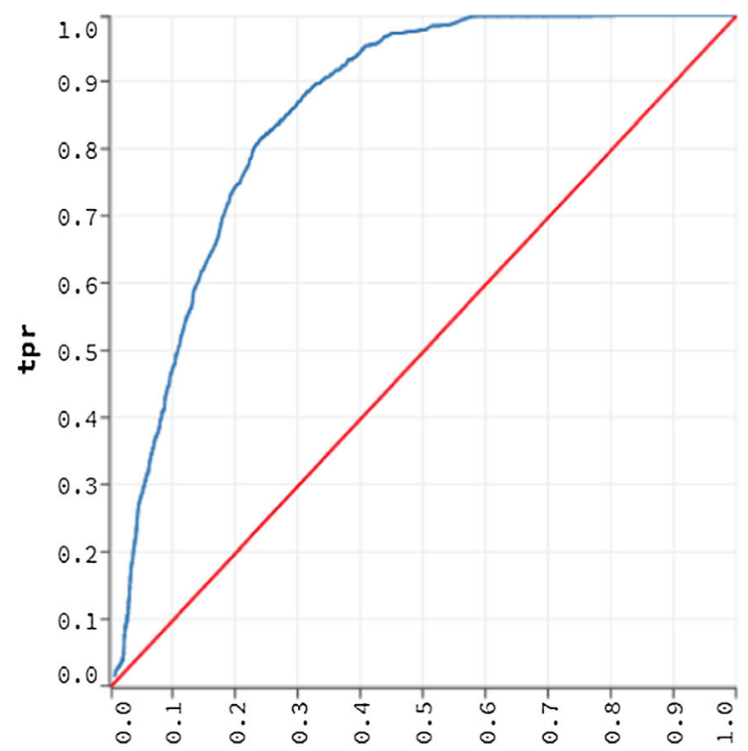

(a) Results for imbalanced dataset

$$
\mathrm{AUC}=0.855
$$

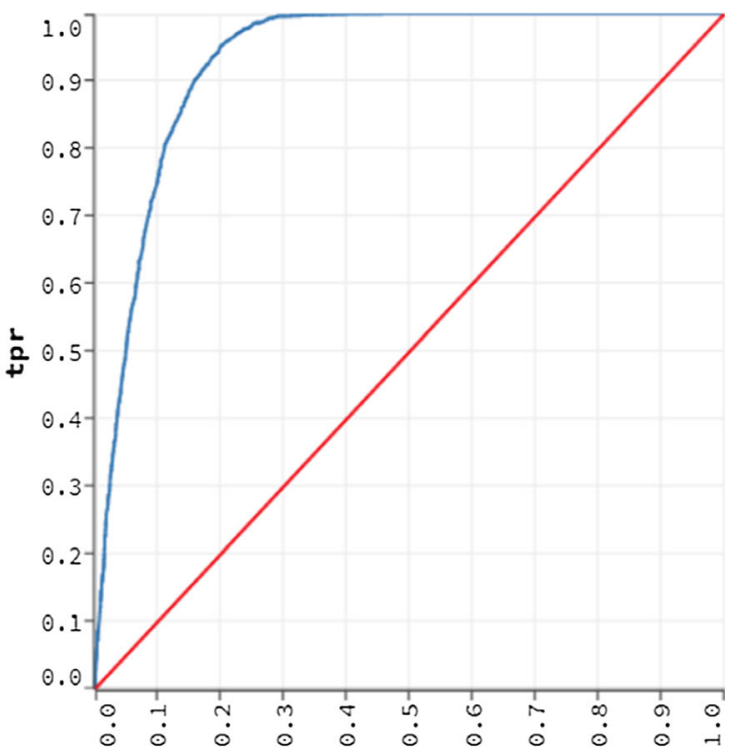

(b) Results for balanced dataset

$$
\mathrm{AUC}=0.932
$$

Fig. 3 ROC Curves for application to a imbalanced and $\mathbf{b}$ balanced datasets. ROC curves plot the true positive rate (tpr) against the false positive rate (fpr)

proposed multi-level deep learning prediction approach to improve the prediction of rare, but important, events in a business process.

\section{Discussion and Conclusion}

This paper investigated the effectiveness of a stacked autoencoders based deep learning approach for predicting future process events of a running process instance. It is the first application of this approach in the business process prediction domain. To evaluate the predictive performance of our method, we compared it against three recent approaches, two of which used LSTM recurrent neural networks, and conventional classification algorithms. Before applying the deep learning model, we used n-gram encoding and feature hashing to build numerical feature vectors from categorical process event data using a sliding window technique. The research objective was to examine the feasibility and impact of applying the proposed approach to process prediction. The experimental results suggest that the proposed method achieves good results on different evaluation metrics and outperforms the state-ofthe-art approaches in most experiments. We also investigated and discussed the effect of adjusting the hyperparameter of the data pre-processing stage and the deep neural networks on the prediction results and applied hyperparameter optimization to find the optimal configuration. Finally, we addressed the imbalanced classification problem by employing neural-network based resampling methods.

In addition to its superior predictive performance, the proposed deep learning approach offers other advantages over conventional techniques. Unsupervised pre-training of neural networks with stacked autoencoders is useful in the presence of unlabeled data as it is able to learn a good feature representation from unlabeled data in a self-supervised manner. Moreover, undercomplete (a deep neural networks architecture with decreasing width (layer sizes) hidden layers) stacked autoencoders can construct useful higher-level feature representations in their layers and thus reduce the feature dimensionality. These higher-level feature representations are useful for other problems in the business process management domain that rely on event trace features, such as case based reasoning problems, process instance similarity search, process instance clustering, process instance retrieval, etc.

Another contribution of this study is the investigation of the advantages provided by data-preprocessing. We have shown the importance of n-grams based encoding of the sequential business process data. In contrast, the majority of prior approaches use simple indexing, which ignores sequential interdependencies among the events and results in relatively low classification performance. We have also shown the importance of data reduction techniques such as feature hashing, which significantly accelerate the 
classification algorithms. This is particularly important for real-time predictions required for automating process decisions.

This study deals with predicting the next activities in the given process trace. Knowing the occurrence probabilities of the next events allows decision makers to dynamically optimize process execution by adjusting resource allocation, rescheduling process activities, changing activities or taking appropriate actions outside the process instance. Alternatively, by predicting the occurrence of an undesired event, the system warns managers and allows them to avoid it proactively.

The successful application of our proposed approach to next event prediction opens up interesting and important avenues for future work. Our method can also be applied to predicting business process outcomes, such as compliance with service-level agreements, process success or failure. Even if there is no need for algorithmic changes, business process outcome prediction requires intensive feature processing work. Using stacked denoising, contractive or other regularized autoencoders may improve the pre-training results over the ones used here, and is also a subject of future research. Finally, applying the proposed multi-stage deep learning approach to regression problems, such as time to next event or remaining time to case completion, is another interesting research question. By combining the next event prediction with other process driven analytics such as activity duration estimation, it is possible to address more complicated decision tasks.

Our approach, like other predictive methods, assumes that the process (and its event log data) is in a steady state. This means that the relationship between input and output does not change over time and a model trained with historical data can be used to predict new data instances. The results on our experimental datasets show this assumption to be valid at least for these datasets. However, it is reasonable to believe that changes may occur in the controlflow (behavioral), resource and data perspectives of the business processes (Bose et al. 2011). Referred also to as concept drift, these changes may be recurring, sudden, gradual and incremental (Bose et al. 2011). It is important to consider this issue when designing and training a model for predictive business process monitoring. In the data preparation phase of our approach, we already adopt the sliding windows technique for creating the dataset to train our algorithm. With a slight adjustment to the current training procedure, e.g., by forgetting old data upon the arrival new data instances (fixed window approach) and retraining the proposed model iteratively, we can obtain a basic model that is able to handle concept drift. In future work, we intend to address feature drift, concept drift, and changing prior distributions by combining our deep learning approach with methods that handle these different aspects of concept drift.

Finally, expert knowledge and relevant cost information is an important issue when evaluating the performance of classifiers. Evaluating whether an accuracy of $80 \%$ (as in BPI 2012 case in this study) is reasonable for adopting the classification models depends on several factors such as the characteristics of application domain, the nature of underlying process, and the legal and financial consequences of misclassification. Classification in this context is a decision making process which combines predictions with utility/cost functions to attain the goal defined by analysts. Different decision makers have different risk tolerances and consequently different utility functions. Hence, the utility functions determine the acceptable performance of probabilistic machine learning approaches. In future research, we aim to integrate our predictive analytics approach with the decision making process in a real world use-case.

We also aim to provide post hoc explanations to address the "black-box" nature of deep-learning methods and make their predictions interpretable to domain experts. This is important to establish trust in the model results. Incorporating automated decision making for process monitoring in EIS (e.g., triggering alerts upon detection of process execution) requires an understanding of the underlying model, as does using the predictive analytics based on PAIS data for decision making in knowledge intensive processes where the humans are the final decision makers. Our future research aims make process prediction models more understandable.

\section{References}

Barga R, Fontama V, Tok WH, Cabrera-Cordon L (2015) Predictive analytics with Microsoft Azure machine learning. Apress, Berkely, CA

Bergstra JS, Bardenet R, Bengio Y, Kégl B (2011) Algorithms for hyper-parameter optimization. Advances in neural information processing systems. Granada, Spain, pp 2546-2554

Bergstra J, Bengio Y (2012) Random search for hyper-parameter optimization. J Mach Learn Res 13(1):281-305

Bose RPJC, van der Aalst WMP, Žliobaitė I, Pechenizkiy M (2011) Handling concept drift in process mining. In: International conference on advanced information systems engineering, Springer, London, pp 391-405

Bradley AP (1997) The use of the area under the ROC curve in the evaluation of machine learning algorithms. Pattern Recognit 30(7):1145-1159

Breuker D, Matzner M, Delfmann P, Becker J (2016) Comprehensible predictive models for business processes. MIS Q 40(4):1009-1034

Candel A, Parmar V, LeDell E, Arora A (2016) Deep learning with h2o. $\mathrm{H} 2 \mathrm{O}$ Inc, CA 
Caragea C, Silvescu A, Mitra P (2012) Protein sequence classification using feature hashing. Proteome Sci 10(1):1-14

Caruana R, Karampatziakis N, Yessenalina A (2008) An empirical evaluation of supervised learning in high dimensions. In: 25th international conference on machine learning, ACM, Helsinki, pp 96-103

Caruana R, Niculescu-Mizil A (2006) An empirical comparison of supervised learning algorithms. In: 23rd international conference on machine learning. ACM, Pittsburgh, pp 161-168

Da Silva NFF, Hruschka ER, Hruschka ER (2014) Tweet sentiment analysis with classifier ensembles. Decis Support Syst 66:170-179

Davenport TH, Harris JG (2007) Competing on analytics: the new science of winning. Harvard Business School Press, Boston

Di Francescomarino C, Dumas M, Federici M, et al (2016) Predictive business process monitoring framework with hyperparameter optimization. In: 28th international conference on advanced information systems engineering, Springer, Ljubljana, pp 361-376

Duan L, Da Xu L (2012) Business intelligence for enterprise systems: a survey. IEEE Trans Ind Inform 8(3):679-687

Erhan D, Bengio Y, Courville A et al (2010) Why does unsupervised pre-training help deep learning? J Mach Learn Res 11:625-660

Evermann J, Rehse J-R, Fettke P (2017) Predicting process behaviour using deep learning. Decis Support Syst 100:129-140

Folino F, Guarascio M, Pontieri L (2012) Discovering context-aware models for predicting business process performances. In: OTM confederated international conferences "on the move to meaningful internet systems", Springer, Rome, pp 287-304

Forman G, Kirshenbaum E (2008) Extremely fast text feature extraction for classification and indexing. In: 17th ACM conference on information and knowledge management. ACM, Napa Valley, pp 1221-1230

Ganchev K, Dredze M (2008) Small statistical models by random feature mixing. In: the ACL08 HLT workshop on mobile language processing, Columbus, $\mathrm{OH}, \mathrm{pp}$ 19-20

Goodfellow IJ, Warde-Farley D, Mirza M, et al (2013) Maxout networks. (preprint) arXiv arXiv:1302.4389. Accessed 30 Oct 2017

Gregor S, Hevner AR (2013) Positioning and presenting design science research for maximum impact. MIS Q 37(2):337-356

Hall M, Frank E, Holmes G et al (2009) The WEKA data mining software: an update. ACM SIGKDD Explor Newslett 11(1):10-18

Han H, Wang W-Y, Mao B-H (2005) Borderline-SMOTE: a new over-sampling method in imbalanced data sets learning. In: Advances in Intelligent Computing. ICIC 2005. Lecture Notes in Computer Science, vol 3644. Springer, Berlin, Heidelberg, pp 878-887

Hinton GE, Osindero S, Teh Y-W (2006) A fast learning algorithm for deep belief nets. Neural Comput 18(7):1527-1554

Hinton GE, Salakhutdinov RR (2006) Reducing the dimensionality of data with neural networks. Science 313(5786):504-507

Huang C, Li Y, Change Loy C, Tang X (2016) Learning deep representation for imbalanced classification. In: IEEE conference on computer vision and pattern recognition, IEEE, Las Vegas, pp 5375-5384

Izadyyazdanabadi M, Belykh E, Mooney M et al (2017) Convolutional neural networks: ensemble modeling, fine-tuning and unsupervised semantic localization for intraoperative CLE images. https://arxiv.org/pdf/1709.03028. Accessed 30 Oct 2017

Kang B, Kim D, Kang S (2012a) Periodic performance prediction for real-time business process monitoring. Ind Manag Data Syst 112(1):4-23
Kang B, Kim D, Kang S-H (2012b) Real-time business process monitoring method for prediction of abnormal termination using KNNI-based LOF prediction. Expert Syst Appl 39(5):6061-6068

Lakshmanan GT, Shamsi D, Doganata YN et al (2015) A markov prediction model for data-driven semi-structured business processes. Knowl Inf Syst 42(1):97-126

Langford J, Li L, Strehl A (2007) Vowpal wabbit online learning project. Technical report, Yahoo!

Larochelle H, Erhan D, Courville A et al (2007) An empirical evaluation of deep architectures on problems with many factors of variation. In: 24th international conference on machine larning, ACM, Corvallis, pp 473-480

LaValle S, Lesser E, Shockley R et al (2011) Big data, analytics and the path from insights to value. MIT Sloan Manag Rev 52:21-32

Le M, Gabrys B, Nauck D (2017) A hybrid model for business process event and outcome prediction. Expert Syst 34(5):e12079

Le M, Nauck D, Gabrys B, Martin T (2014) Sequential clustering for event sequences and its impact on next process step prediction. In: International conference on information processing and management of uncertainty in knowledge-based systems, Springer, Cádiz, pp 168-178

LeCun YA, Bottou L, Orr GB, Müller KR (2012) Efficient backprop. Neural networks: tricks of the trade. Springer, Berlin, pp 9-50

Leontjeva A, Conforti R, Di Francescomarino C, et al (2015) Complex symbolic sequence encodings for predictive monitoring of business processes. In: International conference on business process management, Springer, Innsbruck, pp 297-313

Márquez-Chamorro AE, Resinas M, Ruiz-Cortés A, Toro M (2017) Run-time prediction of business process indicators using evolutionary decision rules. Expert Syst Appl 87:1-14

Mehdiyev N, Evermann J, Fettke P (2017) A multi-stage deep learning approach for business process event prediction. In: IEEE 19th conference on business informatics, IEEE, Thessaloniki, pp 119-128

Metzger A, Leitner P, Ivanovic D et al (2015) Comparing and combining predictive business process monitoring techniques. IEEE Trans Syst, Man, Cybern Syst 45(2):276-290

Polato M, Sperduti A, Burattin A, de Leoni M (2016) Time and activity sequence prediction of business process instances. http:// arxiv.org/abs/1602.07566. Accessed 01 Sept 2017

Robnik-Šikonja M (2014) Data generator based on RBF network. (preprint) arXiv arXiv:1403.7308. Accessed 01 Sept 2017

Rogge-Solti A, Weske M (2013) Prediction of remaining service execution time using stochastic petri nets with arbitrary firing delays. In: International conference on service-oriented computing, Springer, Berlin, pp 389-403

Schmidhuber J (2015) Deep learning in neural networks: an overview. Neural Netw 61:85-117

Senderovich A, Di Francescomarino C, Ghidini C et al (2017) Intra and inter-case features in predictive process monitoring: a tale of two dimensions. In: International conference on business process management, Springer, Barcelona, pp 306-323

Shi S, Chu X (2017) Speeding up convolutional neural networks by exploiting the sparsity of rectifier units. https://arxiv.org/pdf/ 1704.07724. Accessed 30 Oct 2017

Steeman W (2013) BPI challenge 2013. https://doi.org/10.4121/uuid: a7ce5c55-03a7-4583-b855-98b86e1a2b07. Accessed 01 Sept 2017

Sun Z, Pambel F, Wang F (2015) Incorporating big data analytics into enterprise information systems. In: Information and communication technology: third IFIP TC 5/8 international conference, ICT-EurAsia 2015, and 9th IFIP WG 8.9 working conference, CONFENIS 2015, Springer, Daejeon, pp 300-309

Sun Y, Wong AKC, Kamel MS (2009) Classification of imbalanced data: a review. Int J Pattern Recognit Artif Intell 23(4):687-719 
Tax N, Verenich I, La Rosa M, Dumas M (2017) Predictive business process monitoring with LSTM neural networks. In: International conference on advanced information systems engineering, Springer, Essen, pp 477-492

Tomović A, Janičić P, Kešelj V (2006) n-Gram-based classification and unsupervised hierarchical clustering of genome sequences. Comput Methods Programs Biomed 81(2):137-153

Tu JV (1996) Advantages and disadvantages of using artificial neural networks versus logistic regression for predicting medical outcomes. J Clin Epidemiol 49(11):1225-1231

Unuvar M, Lakshmanan GT, Doganata YN (2016) Leveraging path information to generate predictions for parallel business processes. Knowl Inf Syst 47(2):433-461

van der Aalst WMP, Schonenberg MH, Song M (2011) Time prediction based on process mining. Inf Syst 36(2):450-475

van Dongen BF (2012) BPI challenge 2012. https://doi.org/10.4121/ uuid:3926db30-f712-4394-aebc-75976070e91f. Accessed 01 Sept 2017

van Dongen BF, Crooy RA, van der Aalst WMP (2008) Cycle time prediction: when will this case finally be finished? In: OTM confederated international conferences "on the move to meaningful internet systems", Springer, Monterey, pp 319-336

Verenich I (2016) Helpdesk. https://doi.org/10.17632/39bp3vv62t.1. Accessed 01 Sept 2017

Vincent P, Larochelle H, Bengio Y, Manzagol P-A (2008) Extracting and composing robust features with denoising autoencoders. In: 25th international conference on machine learning, ACM, Helsinki, pp 1096-1103

Vincent P, Larochelle H, Lajoie I et al (2010) Stacked denoising autoencoders: learning useful representations in a deep network with a local denoising criterion. J Mach Learn Res 11:3371-3408

Wang S, Yao X (2012) Multiclass imbalance problems: analysis and potential solutions. IEEE Trans Syst Man Cybern Part B (Cybernetics) 42(4):1119-1130

Weinberger K, Dasgupta A, Langford J, et al (2009) Feature hashing for large scale multitask learning. In: Proceedings of the 26th annual international conference on machine learning - ICML'09, ACM, Montreal, pp 1-8

Wickham H, Francois R (2015) dplyr: a grammar of data manipulation. R Package Version 04(1):20

Witt N, Seifert C (2017) Understanding the influence of hyperparameters on text embeddings for text classification tasks. In: International conference on theory and practice of digital libraries, Springer, Thessaloniki, pp 193-204

Wu X, Kumar V, Quinlan JR et al (2008) Top 10 algorithms in data mining. Knowl Inf Syst 14(1):1-37

XES Standard (2016) 1849-2016-IEEE standard for eXtensible event stream (XES) for achieving interoperability in event logs and event streams. http://www.xes-standard.org/. Accessed 01 Sept 2017 